\title{
Determination of plastic properties by instrumented spherical indentation: Expanding cavity model and similarity solution approach
}

\author{
Peng Jiang, Taihua Zhang, ${ }^{\text {a) }}$ Yihui Feng, Rong Yang, and Naigang Liang \\ State Key Laboratory of Nonlinear Mechanics, Institute of Mechanics, Chinese Academy of Sciences, \\ Beijing 100190, China
}

(Received 25 July 2008; accepted 12 November 2008)

\begin{abstract}
The present paper aims to develop a robust spherical indentation-based method to extract material plastic properties. For this purpose, a new consideration of piling-up effect is incorporated into the expanding cavity model; an extensive numerical study on the similarity solution has also been performed. As a consequence, two semi-theoretical relations between the indentation response and material plastic properties are derived, with which plastic properties of materials can be identified from a single instrumented spherical indentation curve, the advantage being that this approach no longer needs estimations of contact radius with given elastic modulus. Moreover, the inconvenience in using multiple indenters with different tip angles can be avoided. Comprehensive sensitivity analyses show that the present algorithm is reliable. Also, by experimental verification performed on three typical materials, good agreement of the material properties between those obtained from the reverse algorithm and experimental data is obtained.
\end{abstract}

\section{INTRODUCTION}

In recent studies, interest has been intensifying in the development of indentation-based methods to extract material elastic-plastic properties. In those studies, the elastic-Hollomon power-law hardening hypothesis was generally adopted, in which the uniaxial true stress-true strain $(\sigma-\varepsilon)$ curves of materials are assumed to be expressible in the form

$$
\left\{\begin{array}{ll}
\sigma=E \varepsilon, & \text { for } \varepsilon \leq \varepsilon_{y} \\
\sigma=E \varepsilon_{y}^{1-n} \varepsilon^{n}, & \text { for } \varepsilon \geq \varepsilon_{\mathrm{y}}
\end{array},\right.
$$

where $E$ is the elastic modulus, $\sigma_{y}$ is the yield stress, $n$ is the strain-hardening exponent, and $\varepsilon_{y}$ is equal to the ratio of yield stress to elastic modulus. This material model has also been used in the present study, and the indenter was assumed to be rigid.

In recent years, some investigations have shown that the stress-strain curve cannot be uniquely determined from loading and unloading curve produced by a single sharptipped indenter. ${ }^{1,2}$ Subsequently, several methods using multiple sharp-tipped indenters have been developed to extract plastic properties, ${ }^{3-8}$ although these methods still present a degree of inconvenience when operating with these indenters. In changing to spherical indentation, various authors have demonstrated that, by analyzing the force-depth curves, it is possible to determine material plastic properties. ${ }^{9-24}$ However, most of these proposed

\footnotetext{
a) Address all correspondence to this author.

e-mail: zhangth@lnm.imech.ac.cn

DOI: 10.1557/JMR.2009.0108
}

methods depend on estimates of the contact area under the spherical indenter tip, ${ }^{9-19}$ the estimates being difficult to obtain especially when "piling-up" occurs. For some methods, additional problems are created by repetitive loading and unloading, such as the method proposed by Kucharski and $\mathrm{Mroz}^{17}$ and that by Huber and Tsakmkis. ${ }^{23}$ Later, the work of Nayebi et al. ${ }^{24}$ demonstrated the feasibility in determining plastic properties by using a single spherical indentation curve, but it is only applicable to steels. In a recent study, by extending the representative strain (as defined in the work of Dao et al. ${ }^{25}$ for sharp indentation) to spherical indentation, two representative methods were developed to avoid the above-mentioned difficulties. $^{20,21}$ Unfortunately, in those two methods, just as in most previous work, the direct sensitivity analysis of the acquired plastic properties $\left(\sigma_{y}\right.$ and $\left.n\right)$, associated with errors in the measurement of indentation parameters, was not mentioned, but it is now recognized as an important factor in evaluating indentation-based methods. Perhaps due to this sensitivity issue, Herbert et al.' ${ }^{26}$ intentions to obtain experimental verification for Al 6061 through the use of a spherical indenter with $385 \mathrm{~nm}$ tip radius, have shown that some of the proposed methods cannot derive reasonable results of $\sigma_{y}$ and $n$.

In our opinion, there are two main reasons for the sensitivity problem of these methods. The first, and the more important of the two, is the ill-formed conditions of the equations used, which stem mainly from the dependence of response parameters. For example, the relation between the ratio of unloading work to total work $W_{u} / W_{t}$ and the ratio of residual depth to max depth $h_{r} / h_{m}$ is expressed in an approximation as $W_{u} / W_{t} \approx h_{r} / h_{m}$ for 
spherical indentation. ${ }^{27}$ Therefore, if these two response parameters $\left(W_{u} / W_{t}, h_{r} / h_{m}\right)$ are used, the sensitivity problem will inevitably arise. The second is that the variation of plastic properties cannot be sufficiently reflected by the selected response parameters, which can also lead to sensitivity issues. According to our numerical results corresponding to $h / R=0.3$ (see Sec. II), the dimensionless load $F / E R^{2}$ of two different combinations of plastic properties $\left(n=0 \quad \varepsilon_{y}=0.025\right.$ and $\left.n=0 \quad \varepsilon_{y}=0.03\right)$ are 0.16 and 0.17 , respectively. It can be easily deduced that, in the reverse analysis, a variation of no more than $6 \%$ in the dimensionless load will lead to more than $15 \%$ variation of $\varepsilon_{y}$.

Therefore, the focus of this study is to determine ways to select the response parameters and to establish the relations between the selected response parameters and plastic properties. According to the above criteria, there are two mandatory requirements in the choice of response parameters: aside from the fact that the selected parameters should be able to adequately reflect the variation of plastic properties, the existence of sufficient independence between these selected response parameters is more important. In the present article, the total work $W_{t}$ and the Meyer index $m$ were selected for this purpose, and to establish relations between these two parameters and the plastic properties, the expanding cavity models $(\mathrm{ECMs})^{28-30}$ and the similarity solution ${ }^{31}$ were used.

By considering the effect of piling-up on the volume displaced by hydrostatic core, a modified ECM, in the present study, was developed to describe the relationship between indentation work and plastic properties. In tandem, the similarity solution was further investigated in detail numerically to obtain another function. From these relations together with the well known elastic modulus extraction method, ${ }^{32}$ a spherical indentation-based method to determine the plastic properties of materials has been proposed.

\section{FORWARD ANALYSIS}

\section{A. Expanding cavity models}

Based on Hill's solution for the quasistatic expansion of an internally pressurized spherical shell of an elasticplastic material, ECMs have been developed to describe the indentation responses of various materials. ${ }^{28,29}$ It is worth noting that such ECMs have been found to break down for materials having significant strain-hardening characteristics. By assuming material incompressibility (specifically a Poisson ratio of $v=0.5$ ), Gao et al. ${ }^{30}$ later developed a new ECM for power-law hardening materials. However, because nonlinear terms had been ignored in estimating the radius of elastic-plastic interface $(c$ in Fig. 1), this ECM has applicability only for small indentation depths $(a \ll R)$. In such cases, plastic properties cannot be significantly characterized by the spherical indentation response parameters, especially for materials with small ratios of elastic modulus to yield stress. ${ }^{30}$ Furthermore, neglecting the piling-up effect also leads to errors in evaluating the volume radially displaced by the hydrostatic core, the investigation of which will be given in detail below.

Following an early suggestion by Johnson, ${ }^{29}$ the stress and displacement outside the hydrostatic core are radially symmetric, which leaves only the radial displacement component $u_{r}$, along with the constraint relations of $\sigma_{\theta}=\sigma_{\phi}$ and $\varepsilon_{\theta}=\varepsilon_{\phi}$, as degrees of freedom for the process. Here $r, \theta$, and $\phi$ denote the coordinates in the spherical coordinate system. Assuming incompressibility of the material, $u_{r}$ can be taken into account by a radially expanded hydrostatic core, thereby deriving its volume as

$$
V=\frac{2}{3} \pi\left[\left(r+u_{r}\right)^{3}-r^{3}\right]=\frac{2}{3} \pi u_{r}\left[3 r^{2}+3 r u_{r}+u_{r}^{2}\right] .
$$

If no piling-up or sinking-in occurs, $V$ should be equal to the volume of indenter tip under the original surface, which can be expressed as

$$
V^{\prime}=\frac{1}{3} \pi h^{2}(3 R-h)
$$

where $R$ and $h$ are the radius of the spherical indenter tip and the indentation depth, respectively.

However, when piling-up or sinking-in occurs, some fraction of the compressed material is ejected or compacted into original surface, the volume of which is denoted as $V_{p}$ or $V_{s}$ in Fig. 1. Therefore, the volume of material that is radially expanded by the hydrostatic core will differ. A great simplification can be derived if the volumes $V_{p}$ and $V_{s}$ are assumed to be some fraction of the hydrostatic core volume displaced by the indenter tip, here denoted by $V_{p}^{\prime}$ and $V_{s}^{\prime}$ around the indenter in Fig. 1. In this way, we introduce a correction factor $\kappa$ to take into account this piling-up effect on $V$ as shown below

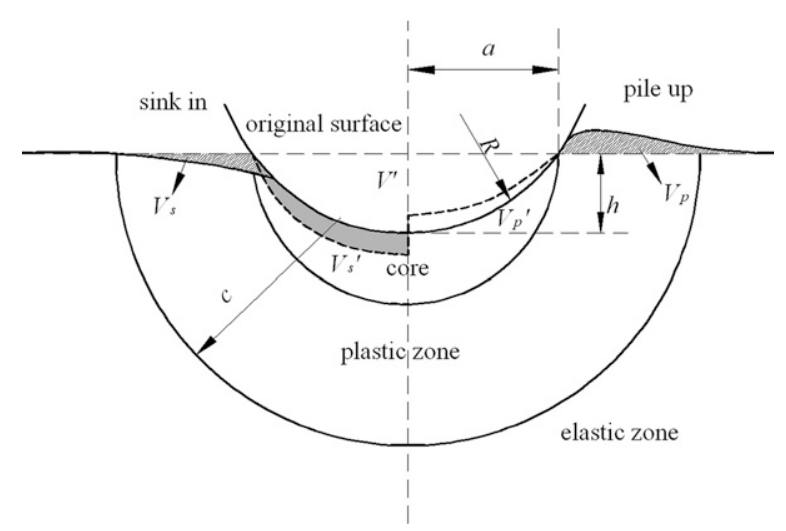

FIG. 1. Schematic of the modified expanding cavity model in which the effect of piling-up or sinking-in is equivalent to some fraction of hydrostatic core volume displaced by indenter. 


$$
V=\kappa^{3} V^{\prime}=\frac{1}{3} \kappa^{3} \pi h^{2}(3 R-h)
$$

Assuming small deformations $\left(u_{r} \ll r\right)$, we can derive $u_{r}$ from Eq. (2) as

$$
u_{r} \approx \frac{V}{2 \pi r^{2}}
$$

and the constitutive equation ${ }^{33}$ with Eq. (5) then gives

$$
\varepsilon_{r}=-2 \varepsilon_{\theta}=-\frac{2 u_{r}}{r} \approx-\frac{V}{\pi r^{3}}
$$

By symmetry considerations, the state of stress is everywhere just hydrostatic tension $\sigma_{\theta}$ supposed a radial uniaxial stress $\sigma_{\theta}-\sigma_{r}{ }^{33}$ Because there is no additional elastic strain produced by the hydrostatic tension $(v=0.5)$, the relationship between the uniaxial stress $\left|\sigma_{\theta}-\sigma_{r}\right|$ and strain $\left|\varepsilon_{r}\right|$ can be directly obtained by applying uniaxial test data. ${ }^{33}$ For power-law hardening materials, the relation between $\left|\sigma_{\theta}-\sigma_{r}\right|$ and $\left|\varepsilon_{r}\right|$ can be expressed as ${ }^{30}$

$$
\left\{\begin{array}{ll}
\left|\sigma_{r}-\sigma_{\theta}\right|=E\left|\varepsilon_{r}\right|, & \text { for }\left|\varepsilon_{r}\right| \leq \varepsilon_{y} \\
\left|\sigma_{r}-\sigma_{\theta}\right|=E \varepsilon_{y}^{1-n}\left|\varepsilon_{r}\right|^{n}, & \text { for }\left|\varepsilon_{r}\right| \geq \varepsilon_{y}
\end{array} .\right.
$$

Therefore, $\left|\varepsilon_{r}\right|$ on the elastic-plastic boundary should satisfy

$$
\left|\varepsilon_{r}\right|_{r=c}=\frac{V}{\pi c^{3}}=\varepsilon_{y} .
$$

Combining Eq. (4) and Eq. (8) gives

$$
c=\sqrt[3]{\frac{V}{\pi \varepsilon_{y}}=\kappa \sqrt[3]{\frac{h^{2}(3 R-h)}{3 \varepsilon_{y}}} .}
$$

It has already been remarked that each element of the hemispherical shell is stressed, apart from a supposed hydrostatic tension $\sigma_{\theta}$, by an uniaxial amount $\left|\sigma_{r}-\sigma_{\theta}\right|$. It is evident that the hydrostatic stress does not produce any strain energy $(v=0.5)$, and hence the strain energy density expended by uniaxial stress $\left|\sigma_{r}-\sigma_{\theta}\right|$ can be expressed by

$$
w=\int\left|\sigma_{r}-\sigma_{\theta}\right| d\left|\varepsilon_{r}\right| .
$$

By inserting Eq. (7) into Eq. (10), the strain energy density of the elastic and elastic-plastic region can be derived as

$$
\left\{\begin{array}{ll}
w_{1}=\frac{n-1}{2(n+1)} E \varepsilon_{y}^{2}+\frac{E \varepsilon_{y}^{1-n}}{n+1} \varepsilon^{n+1} & (c \geq r \geq a) \\
w_{2}=\frac{1}{2} E \varepsilon^{-2} & (r \geq c)
\end{array},\right.
$$

where $a$ is the radius of the hemispherical hydrostatic core, which is easily found to be $a=\sqrt{2 R h-h^{2}}$.
From Eqs. (6) and (8), we can derive the distribution of equivalent strain as

$$
\left|\varepsilon_{r}\right|=\left(\frac{c}{r}\right)^{3} \varepsilon_{y}
$$

Because the friction was found to produce negligible effects on force-depth curves, ${ }^{34}$ the total external work is approximately equal to the total strain energy, which can be written as

$$
\begin{aligned}
W_{t}= & \int_{a}^{c} w_{1} 2 \pi r^{2} d r+\int_{c}^{\infty} w_{2} 2 \pi r^{2} d r \\
= & \int_{a}^{c}\left(\frac{n-1}{2(n+1)} E \varepsilon_{y}^{2}+\frac{E \varepsilon_{y}^{1-n}}{n+1} \varepsilon_{r}^{n+1}\right) 2 \pi r^{2} d r \\
& +\int_{c}^{\infty} \frac{1}{2} E \varepsilon_{r}^{-2} 2 \pi r^{2} d r .
\end{aligned}
$$

It should be noticed that there is only hydrostatic pressure and the material is treated as an incompressible fluid, so there is no work done on the core region. ${ }^{35}$ Inserting Eq. (12) into Eq. (13) leads to the total strain energy, which can be integrated as

$$
\begin{aligned}
W_{t}= & \frac{2 \pi E \varepsilon_{y}^{2} c^{3}}{3 n(n+1)}\left[\left(\frac{c}{a}\right)^{3 n}-1\right]+\frac{(n-1) \pi E \varepsilon_{y}^{2} a^{3}}{3(n+1)} \\
& {\left[\left(\frac{c}{a}\right)^{3}-1\right]+\frac{\pi E \varepsilon_{y}^{2} c^{3}}{3}, }
\end{aligned}
$$

where $c$ can be determined by Eq. (9), and $\kappa$ is the correction factor considering the piling-up effect. According to the results of dimensional analysis of $\mathrm{Ni}$ and Cheng, ${ }^{27}$ the degree of the piling-up or sinking-in depends only on $\varepsilon_{y}$ and $n$ with fixed $h / R$. In the present study, we take $h / R$ $=0.3$, which is sufficient to reflect differences in material plastic properties, and therefore we can assume that

$$
\kappa=f\left(\varepsilon_{y}, n\right) .
$$

To determine the function $f$, finite element calculations were performed using ABAQUS for different combinations of plastic properties, with $\varepsilon_{y}$ from 0.0003 to 0.03 and $n$ from 0 to 0.5 , which cover most engineering metals. The spherical indenter tip was assumed to be rigid. Coulomb's friction law was applied to the contact surfaces, and the friction coefficient was taken to be 0.15 . Poisson's ratio was also fixed at 0.3 . Both of these parameters are minor factors in the indentation analysis. ${ }^{34}$ By setting the total work obtained in the simulations to equal the corresponding analytical ones, values of $\kappa$ (see Fig. 2) can be derived. The fitting function $f$ is given as

$$
\begin{aligned}
\kappa= & f\left(\varepsilon_{y}, n\right)=\left(-0.0077 n^{2}+0.0534 n-0.0304\right) \log ^{2}\left(\varepsilon_{y}\right) \\
& +\left(0.3717 n^{2}-0.1331 n-0.0774\right) \log \left(\varepsilon_{y}\right) \\
& +0.495 n^{2}-0.3016 n+1.0627 .
\end{aligned}
$$

As illustrated in Fig. 2, the value of $\kappa$ for small $\varepsilon_{y}$ can be either greater or smaller than unity and depends on 
the degree of work hardening. For large $\varepsilon_{y}, \kappa$ is greater than one for all values $(n>0)$. According to the above analyses, we can deduce that smaller values of $\kappa$ are obtained with greater piling-up. With different combinations of plastic properties, opposite trends can therefore be observed for values of correction factors $\kappa$ and the degree of piling-up from those obtained by Cheng and Cheng. ${ }^{36}$

It should be stressed that the Poisson ratio, as well as the friction coefficient, adopted in the simulations and theoretical analysis were not identical. However, a previous investigation has shown that such differences will not produce significant effects on force-depth curves, ${ }^{34}$ and even if small effects occur, these have already been included in the correction factor $\kappa$.

Thus, by inserting Eqs. (9) and (16) into Eq. (14), relations between the indentation work $\left(W_{t}\right)$ and plastic properties $\left(\varepsilon_{y}, n\right)$ can be established. The comparison between the indentation work obtained by Eq. (14) and that from simulations is shown in Fig. 3. It is clear that even for various indentation depths $(0.1 R \leq h \leq 0.3 R)$, Eq. (14) can also give an approximate estimation of the total indentation work. Moreover, unlike previous work $^{22}$ in which three sets of 144 empirical parameters are in need, Eq. (14) derived in the present study contains only nine empirical parameters. However, if we use the total work calculated from two different depths to solve for the plastic properties, $\varepsilon_{y}$ and $n$, similar equations used will result in an ill-formed condition for the solution. To derive stable results for plastic properties, we resort to the similarity solution, ${ }^{31}$ in which the Meyer index is mainly dependent on $n$. Moreover, we can deduce from Eq. (14) that $W_{t}$ depends on both $\varepsilon_{y}$ and $n$, so it is reasonable to believe that the issues associated with the ill-formed solution can be resolved to a certain degree with the selection of such parameters.

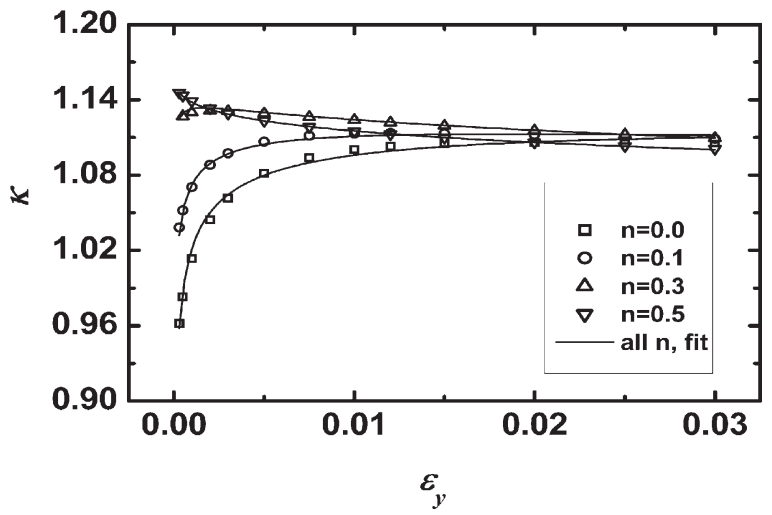

FIG. 2. The relationship between the correction factor $\kappa$ and the plastic properties of materials. The fitting results are calculated by Eq. (16).
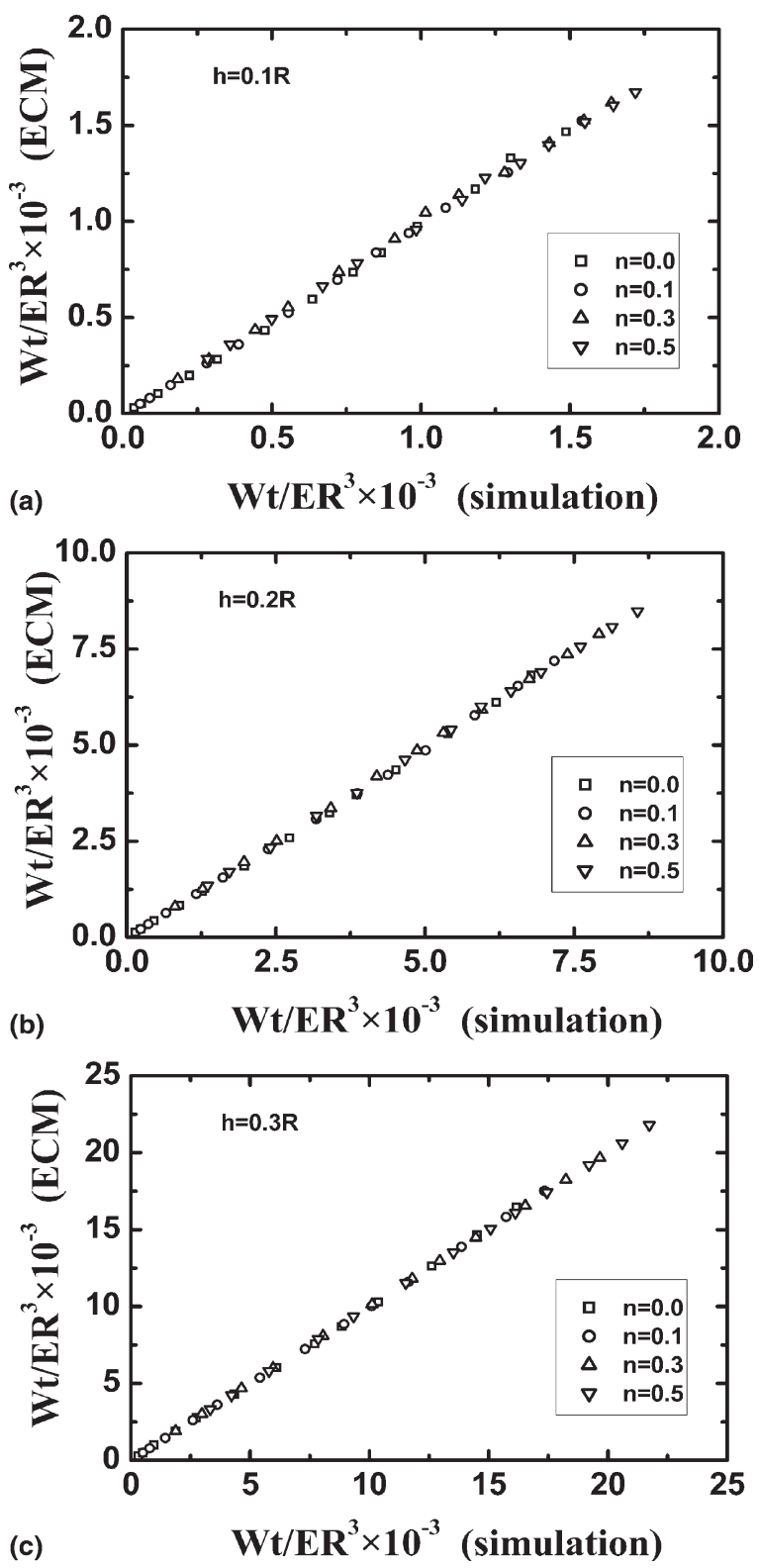

FIG. 3. Comparison between the indentation work obtained by Eq. (14) and the indentation work obtained by simulation: (a) $h / R=$ 0.1 , (b) $h / R=0.2$, and (c) $h / R=0.3$.

\section{B. Similarity solution}

The most extensive studies of spherical indentation were realized by Meyer, ${ }^{37}$ who found that mean pressure increases with the ratio of the size of impression to the spherical diameter according to the simple power law. Subsequently, Hill et al. ${ }^{31}$ demonstrated, in particular for spherical indentation of power-law solids, that solutions for moving contact may be generated from a stationary solution by an appropriate scaling procedure. In this spirit, they provided a rigorous solution, the so-called similarity solution, as follows 


$$
\frac{F}{\pi a_{c}^{2}}=\alpha \beta^{n} k\left(\frac{a_{c}}{2 R}\right)^{n},
$$

where $F$ is the indentation force; $a_{c}$ is the contact radius that is always assumed to be the same as the radius of the residual impression, and $\alpha$ and $\beta$ are universal constants with values $\alpha=2.8, \beta=0.4$ proposed previously by Tabor. ${ }^{9}$ Moreover, according to the suggestion of Hill, the ratio $c_{m}=a_{c} / a$ is an invariant throughout the indentation process and is dependent only on the strain-hardening exponent $n$ as follows $^{31}$

$$
c_{m}^{2}=\frac{5}{2}\left(\frac{2-n}{4+n}\right) .
$$

Inserting Eq. (17) into Eq. (18) and taking logarithms, Field and Swain ${ }^{16}$ obtained

$$
\log F=\log \left(\frac{\alpha \beta^{n} c_{m}^{2+n}}{2^{n} \mathrm{R}^{n}}\right)+(2+n) \log a .
$$

Clearly, a linear regression of $\log F$ and $\log a$ gives $2+n$ as the gradient, which is also called Meyer index $(m)$. However, a more extensive numerical study by Mesarovic and Fleck ${ }^{34}$ has shown that the domain of validity of the similarity solution is limited both by elastic effects for small indentation depth and finite deformation effects for large indentation depth, and they suggested that the similarity solution cannot be directly used to extract the properties of materials.

In the opinion of the present authors, an empirical modification may after all be accepted as a feasible means to develop greater accuracy and expand the domain of validity of the similarity solution. In the present study, a fixed range of $0.6 \leq a / R \leq 0.7$ was chosen so that $h$ is restricted to less than $0.3 R$. Using the extreme points of the range, the gradients of linear regressions of $\log F$ and $\log a$ can be approximately expressed as

$$
m=\frac{\log \left(F_{0.7} / F_{0.6}\right)}{\log (0.7 R / 0.6 R)}=14.9 \log \left(\frac{F_{0.7}}{F_{0.6}}\right),
$$

where $F_{0.6}$ and $F_{0.7}$ are the indentation forces corresponding to $a=0.6 R$ and $a=0.7 R$, respectively. According to the dimensional analysis of Cheng and Cheng, ${ }^{36}$ we can derive

$$
\begin{aligned}
& \frac{F_{0.6}}{E R^{2}}=f_{0.6}\left(v, \varepsilon_{y}, n\right) \\
& \frac{F_{0.7}}{E R^{2}}=f_{0.7}\left(v, \varepsilon_{y}, n\right) .
\end{aligned}
$$

Because the functional dependence on $v$ is found to be small, it can be neglected, and we then obtain from Eq. (20)

$$
m=14.9 \log \left[\frac{f_{0.7}\left(\varepsilon_{y}, n\right)}{f_{0.6}\left(\varepsilon_{y}, n\right)}\right]=f_{m}\left(\varepsilon_{y}, n\right)
$$

Based on the above finite-element method (FEM) results, the gradients of linear regression of $\log F$ and $\log a$ for various materials are shown in Fig. 4. It is obvious that, unlike the suggestion by Field and Swain, ${ }^{16}$ the gradient $m$ not only depends on $n$, but it also slightly depends on $\varepsilon_{y}$. The fitting function of $m$ is given as

$$
\begin{aligned}
m= & \left(-792.59 n^{2}+1675.9 n-962.01\right) \varepsilon_{y}^{2} \\
& +\left(68.187 n^{2}-112.78 n+57.84\right) \varepsilon_{y} \\
& -1.4569 n^{2}+2.8637 n+1.7178 .
\end{aligned}
$$

\section{REVERSE ANALYSIS}

\section{A. The principle of reverse analysis}

As a direct application of the analytical expressions, Eqs. (14) and (23), presented in Sec. II, a new method has been developed for plastic properties characterization as illustrated in Flow Chart 1. For a given spherical indentation test with a specific range of $h / R(h / R$ from 0 to $0.3 R$ ), $W_{t}$ and $m$ can be obtained from the forcedepth curve. Considering the fact that the elastic modulus $E$ of tested materials can be determined by the Oliver-Pharr method ${ }^{32}$ with assumption of $v=0.3, E$ is then considered as a priori known. Using Newton's iteration method, Eqs. (14) and (23) can be solved for plastic parameters $\sigma_{y}\left(\sigma_{y}=E \varepsilon_{y}\right)$ and $n$.

\section{B. Numerical verification}

Numerical indentation simulations were first performed to examine the effectiveness of the reverse analysis algorithm. In this article, materials with different plastic properties within the following ranges, $\varepsilon_{y 0}$ varies from 0.0003 to 0.03 , and $n_{0}$ is set between 0 and 0.5 , were simulated so that most engineering materials are covered. Combinations $\left(\varepsilon_{y 0}, n_{0}\right)$ were inputted in FEM

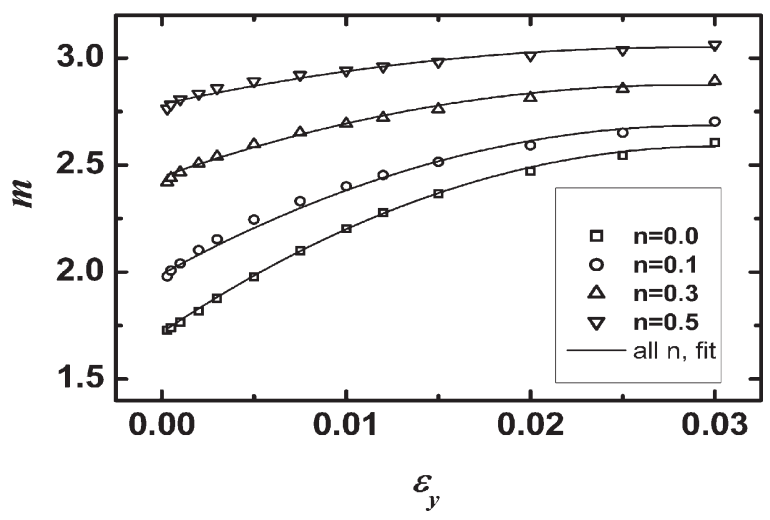

FIG. 4. The relationship between the Meyer index $m$ and material plastic properties. The fitting results are calculated by Eq. (23). 
experiments, and the resultant force-depth curves were then used to calculate indentation parameters $W_{t}$ and $m$, from which the material plastic properties $\left(\varepsilon_{y}, n\right)$ can be derived by our reverse algorithm. The numerical verification results are shown in Table I, where $\varepsilon_{y 0}$ and $n_{0}$ represent the input parameters while $\varepsilon_{y}$ and $n$ are results predicted by reverse analysis. For all cases, it can be readily seen that after several iterations the results converge to unique solutions. Comparing solutions obtained by our method with the input parameters, the maximum error of the new spherical indentation algorithm is approximately $10 \%$, and in most cases these errors are below 5\%, which makes the algorithm fairly reliable with a satisfactory accuracy.

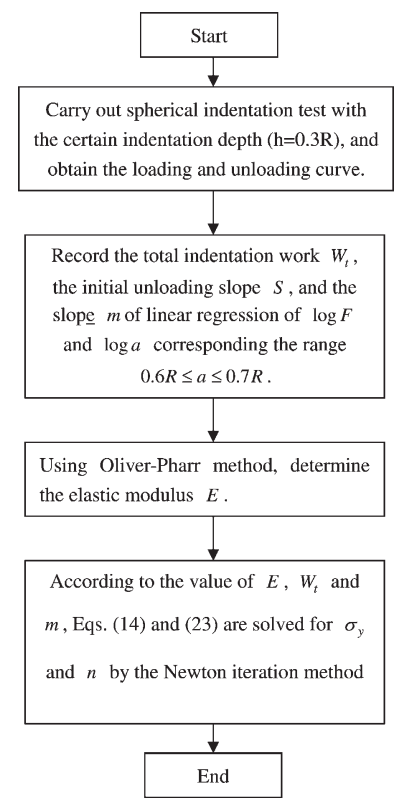

FLOW CHART 1. Extracting plastic properties of materials from spherical indentation tests.
Furthermore, for this indentation-based method to be widely adopted in extracting plastic properties, it must be proven to be sufficiently robust so that small uncertainties or variations in the measured indentation response parameters from experiments, which could arise due to several factors associated with the test instrumentation, the test environment, or test material, do not lead to unreasonable high variability in the estimation of material properties. Therefore, the stability of the proposed method needs to be investigated in greater detail.

To characterize the sensitivity of the proposed method, independent relative perturbations were introduced into $W_{t}, m$, and $E$. Following an early suggestion of $\mathrm{Cao}^{20}$ and Oliver, ${ }^{32}$ the relative perturbation of $E$ to be introduced in the present sensitivity analysis was set to be $4 \%$, and the relative perturbations of $W_{t}$ and $m$ were determined to be 3 and $2 \%$, respectively, according to the discrete degree of physical test data of three typical materials, which are to be presented in the next section. After introducing these perturbation levels in the response parameters, the inverse approach outlined in Flow Chart 1 was followed to identify the plastic properties $\sigma_{y}{ }^{\prime}$ and $n^{\prime}$. The relative errors of yield stresses [i.e., $\left.\left(\sigma_{y}{ }^{\prime}-\sigma_{y 0}\right) / \sigma_{y 0}\right]$ were then presented as three-dimensional maps (see Fig. 5). It can be clearly seen that the maximum deviation of yield strength of the algorithm is approximately $30 \%$, and in most cases these errors are below $10 \%$.

\section{Experimental verification}

All of the indentation experiments were performed using an MTS Nano Indenter XP (Oak Ridge, TN), and the nominal radius of spherical indenter tip was given as $10.6 \mu \mathrm{m}$. To verify the radius of the indenter, Steel IF was selected as the standard reference material. It has a relatively smooth surface and a known true stress-true strain curve. The loading rate was held at the constant

TABLE I. Comparison between the input values and identified plastic properties for various materials simulated in this article.

\begin{tabular}{|c|c|c|c|c|c|c|c|c|}
\hline \multirow[b]{2}{*}{$\varepsilon_{\mathrm{y} 0}(\%)$} & \multicolumn{2}{|c|}{$n_{0}=0$} & \multicolumn{2}{|c|}{$n_{0}=0.1$} & \multicolumn{2}{|c|}{$n_{0}=0.3$} & \multicolumn{2}{|c|}{$n_{0}=0.5$} \\
\hline & $\mathrm{N}$ & $\varepsilon_{\mathrm{y}}(\%)$ & $\mathrm{N}$ & $\varepsilon_{\mathrm{y}}(\%)$ & $\mathrm{N}$ & $\varepsilon_{\mathrm{y}}(\%)$ & $\mathrm{N}$ & $\varepsilon_{\mathrm{y}}(\%)$ \\
\hline 0.0300 & -0.0025 & 0.0307 & 0.0962 & 0.0314 & 0.2886 & 0.0322 & 0.4803 & 0.0312 \\
\hline 0.0500 & -0.0021 & 0.0498 & 0.0976 & 0.0508 & 0.2886 & 0.0529 & 0.4908 & 0.0540 \\
\hline 0.1000 & -0.0028 & 0.0984 & 0.1019 & 0.0985 & 0.2954 & 0.1009 & 0.5030 & 0.0988 \\
\hline 0.2000 & -0.0044 & 0.1984 & 0.1106 & 0.1915 & 0.3029 & 0.1951 & 0.5109 & 0.1899 \\
\hline 0.3000 & -0.0014 & 0.2982 & 0.1154 & 0.2854 & 0.3069 & 0.2891 & 0.5196 & 0.2743 \\
\hline 0.5000 & -0.0039 & 0.5085 & 0.1242 & 0.4706 & 0.3112 & 0.4785 & 0.5234 & 0.4554 \\
\hline 0.7500 & -0.0041 & 0.7681 & 0.1227 & 0.7176 & 0.3101 & 0.7251 & 0.5178 & 0.7047 \\
\hline 1.0000 & -0.0022 & 1.0285 & 0.1149 & 0.9789 & 0.2992 & 0.9991 & 0.5044 & 0.9833 \\
\hline 1.2000 & -0.0032 & 1.2291 & 0.1097 & 1.1879 & 0.2874 & 1.2325 & 0.4972 & 1.2036 \\
\hline 1.5000 & -0.0107 & 1.5456 & 0.0942 & 1.5233 & 0.2753 & 1.5752 & 0.4832 & 1.5537 \\
\hline 2.0000 & -0.0249 & 2.0679 & 0.0717 & 2.0803 & 0.2657 & 2.1198 & 0.4705 & 2.1173 \\
\hline 2.5000 & -0.0171 & 2.5113 & 0.0804 & 2.5357 & 0.2821 & 2.5665 & 0.4762 & 2.6142 \\
\hline 3.0000 & 0.0211 & 2.8466 & 0.1182 & 2.8800 & 0.3161 & 2.9332 & 0.4975 & 3.0406 \\
\hline
\end{tabular}


value of $1 \mathrm{mN} / \mathrm{s}$, and 10 indentation tests were performed on samples of the target material. The average measured force-depth curve was compared with simulated forcedepth curve obtained by introducing in the FE analysis the true stress-true strain data. The indentation dimensionless load $F / E R^{2}$ was plotted versus the dimensionless depth $h / R$. As shown in Fig. 6, both the experimental and the FE-simulated indentation curves are in good agreement, when the tip radius was $10.8 \mu \mathrm{m}$, a value very close to its nominal radius.

Aside from Steel IF, two other typical materials with known uniaxial true stress-true strain relations, Al 6061 and Steel S45C, were chosen to examine the proposed method. Ten experiments were performed for each material. Figure 7 illustrates the average load-displacement data acquired for the three types of materials according to the procedure outlined above. The error bars span one standard deviation about the mean values.
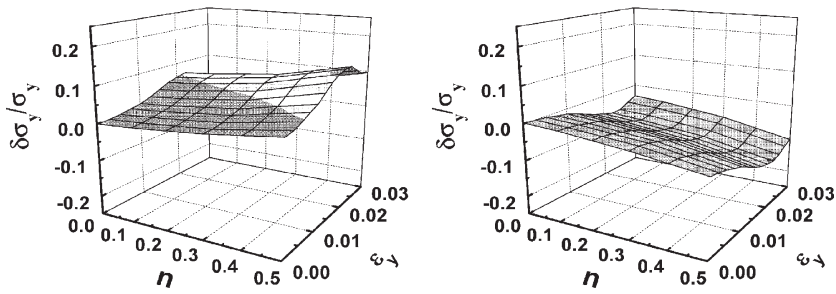

(a)

(b)

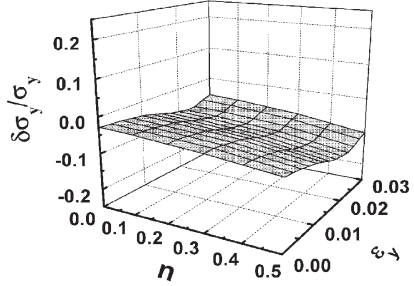

(c)

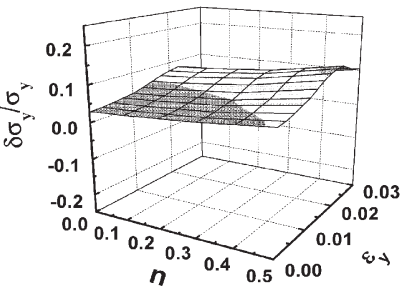

(d)
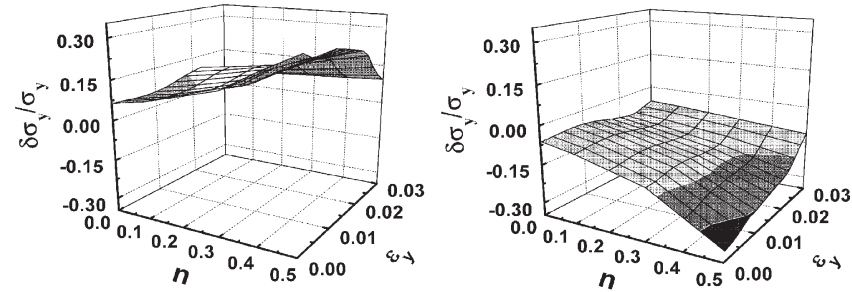

(e)

(f)

FIG. 5. Sensitivity distribution observed in the determination of the yield stress with uncertainties of $(\mathrm{a}, \mathrm{b})+4$ and $-4 \%$ in $E,(\mathrm{c}, \mathrm{d})+3$ and $-3 \%$ in $W_{t}$, and $(\mathrm{e}, \mathrm{f})+2$ and $-2 \%$ in $m$.
Figure 8 illustrates the linear regression of $\log (F)$ versus $\log (a)$. Furthermore, the mean values and coefficients of variation $(\mathrm{COVs})$ of indentation response parameters are presented in Table II, among which the elastic modulus were obtained by the Oliver-Pharr method. ${ }^{32}$ From Table II, it is clear that the COVs of $E, W_{t}$, and $m$ are below $\pm 4, \pm 3$, and $\pm 2 \%$, respectively. It is reasonable to believe that the actual values of $E, W_{t}$, and $m$ should be restricted in the variation range of their physical test data, if enough replications of tests are performed and no obvious faults exist in the test procedures. The above sensitivity analyses were performed just based on this assumption. By introducing the mean values of $E, W_{t}$, and $m, \sigma_{y p}$ and $n_{p}$ of each tested material can be derived by our method. The results, together with the actual true stress-true strain relations obtained by tension tests, are shown in Fig. 9. In addition, the relative errors of yield stress determined by our method are given in Table III,

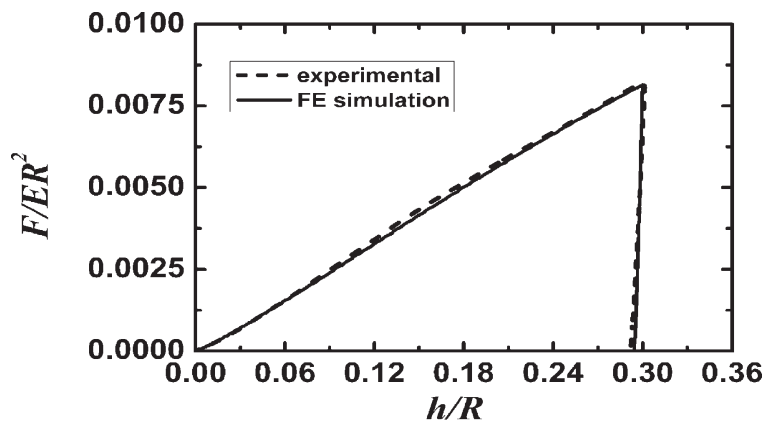

FIG. 6. Comparison between numerical and experimental indentation curves for Steel IF assuming a radius of $10.8 \mu \mathrm{m}$.

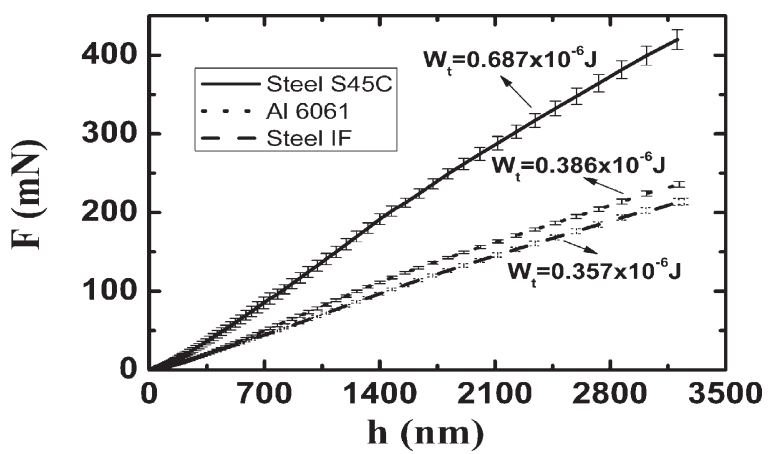

FIG. 7. Averaged load versus normalized displacement curves for Steel S45C, Al 6061, and Steel IF. The error bars span one standard deviation about the mean.

TABLE II. The mean values and COVs of $E, W_{t}$, and $m$ obtained from for three materials.

\begin{tabular}{|c|c|c|c|c|c|c|}
\hline \multirow[b]{2}{*}{ Materials } & \multicolumn{2}{|c|}{ E } & \multicolumn{2}{|c|}{$W_{t}$} & \multicolumn{2}{|c|}{$m$} \\
\hline & Mean (GPa) & $\operatorname{COV}(\%)$ & Mean $\left(J \times 10^{-6}\right)$ & $\operatorname{COV}(\%)$ & Mean & $\operatorname{COV}(\%)$ \\
\hline Steel S45C & 173.89 & \pm 3.2 & 0.687 & \pm 2.1 & 2.142 & \pm 2.3 \\
\hline Al 6061 & 71.23 & \pm 1.9 & 0.386 & \pm 2.3 & 2.136 & \pm 0.9 \\
\hline Steel IF & 188.83 & \pm 3.0 & 0.357 & \pm 2.8 & 2.135 & \pm 1.4 \\
\hline
\end{tabular}




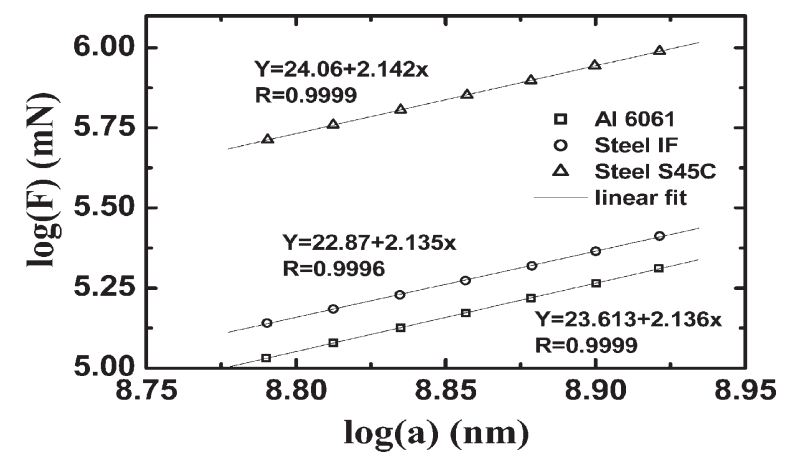

FIG. 8. Linear regression of $\log (\mathrm{F})$ versus $\log$ (a) used in the similarity solution.
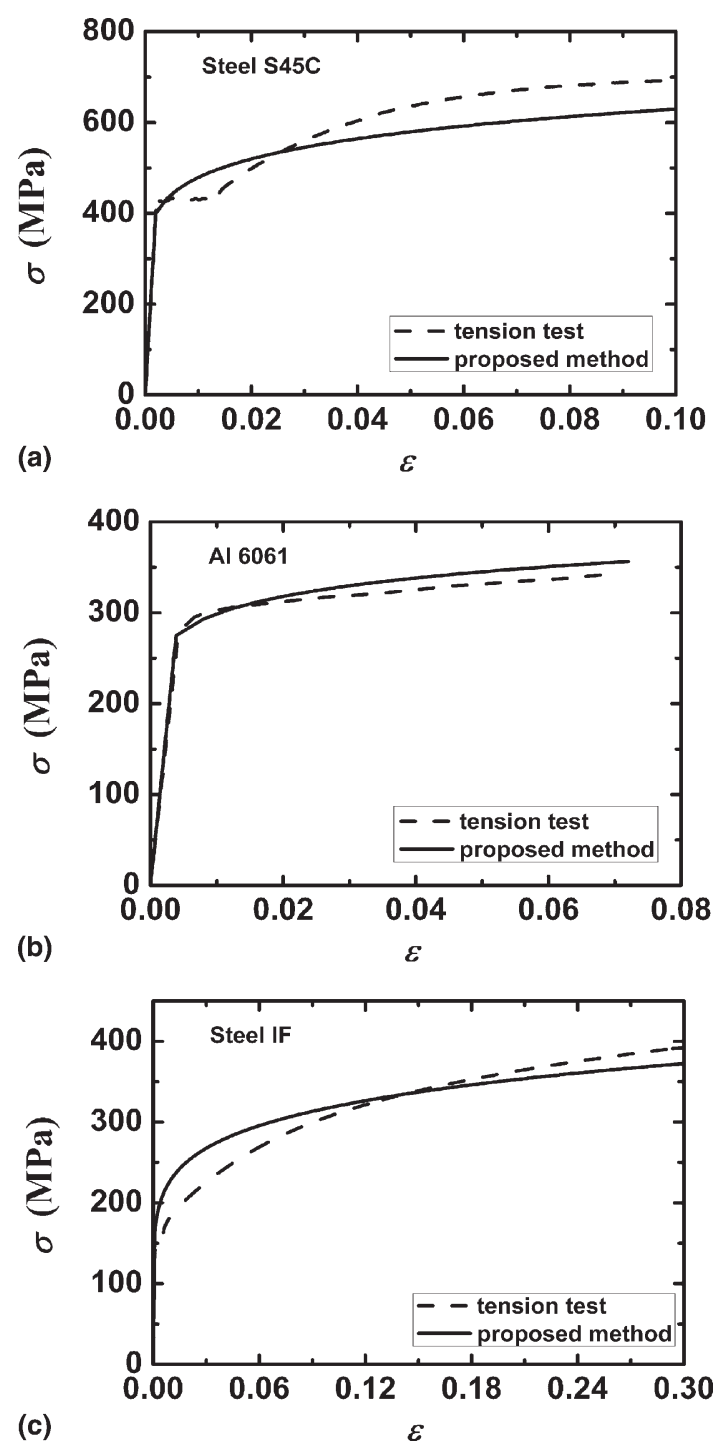

FIG. 9. Comparison between estimated and actual true stress-true strain curves of (a) Steel S45C, (b) Al 6061, and (c) Steel IF.

in which $\sigma_{y t}$ stands for the actual yield strength. From Fig. 9 and Table III, it is evident that the proposed method can give good estimations of the yield strengths and hardening behaviors of practical materials.
TABLE III. Relative errors of the yield strengths estimated by the proposed method.

\begin{tabular}{lccc}
\hline \hline Materials & $\sigma_{y p}(\mathrm{MPa})$ & $\sigma_{y t}(\mathrm{MPa})$ & $\left(\sigma_{y p}-\sigma_{y t}\right) / \sigma_{y t}$ \\
\hline Steel S45C & 400 & 425 & $-5.8 \%$ \\
Al 6061 & 277 & 275 & $-0.7 \%$ \\
Steel IF & 161 & 149 & $8.3 \%$ \\
\hline \hline
\end{tabular}

It is worth noting that the Oliver-Pharr model should be used with caution for a wide range of materials, especially when piling-up occurs. However, from Fig. 5, we can conclude that the proposed method is, fortunately, insensitive to the error in the elastic modulus, so good agreements between estimated and actual results can be derived for most common metals, at least for the materials selected in this article. Furthermore, we can also resort to other methods to extract the elastic modulus, for example, the method proposed by $\mathrm{Ni}$ and Cheng, ${ }^{27}$ in which there is also no need in estimating the contact radius, whereas more experimental verifications are needed.

It must also be stressed that the proposed method is not applicable for very shallow indentation depths, because in such cases, the surface roughness, the grain size of materials, and the indentation seize of effect (ISE) will obviously affect the test results. However, the sensitivity problem of the indentation-based method cannot be avoided completely. Therefore, we suggest that the method proposed in this article should be used with indentation depths of more than $1 \mu \mathrm{m}$.

\section{CONCLUSION}

In this article, theoretical analyses and numerical studies were performed to elucidate the mechanics of instrumented spherical indentation, and a new method has been proposed to extract the plastic properties of materials from only a single indentation curve. The key results of this investigation can be summarized as follows:

(1) By taking into account the effect of piling-up, we developed a modified ECM for power-law hardening materials, which provides us with a semi-theoretical relation between the total indentation work and properties of materials at different depths $(0.1 \leq h / R \leq 0.3)$. Based on the similarity solution, an empirical relation with fixed validity range of $0.6 \leq h / R \leq 0.7$ was also derived.

(2) Using these two relations, a new inverse approach to extract plastic properties of materials from the spherical indentation loading curve has been presented. In contrast to most previous work, the present approach no longer needs the estimation of contact radius or residual radius of indentation with known elastic modulus; also the inconvenience of using multiple indenters with different tip angles is no longer required here.

(3) By considering the divergence of the actual test data, more extensive sensitivity analyses using numerical 
methods were performed for a wide range of materials. Furthermore, three materials with known uniaxial stressstrain relations were selected to examine the practicability of the proposed method. Both numerical and experimental results indicate that the present approach has the ability to effectively measure plastic properties of materials.

\section{ACKNOWLEDGMENTS}

We thank Prof. Dejun Ma, Dr. Xiufang Wang, and Xiaoping Yang for help in providing the uniaxial stressstrain curves of Steel S45C, Al 6061, and Steel IF. We acknowledge the support of the National Natural Science Foundation of China (NSFC) (Grant Nos. 10872200, 10721202, 10572140, and 10232050).

\section{REFERENCES}

1. Y.T. Cheng and C.M. Cheng: Can stress-strain relationships be obtained from indentation curves using conical and pyramidal indenters? J. Mater. Res. 14(9), 3493 (1999).

2. X. Chen, N. Ogasawara, M.H. Zhao, and N. Chiba: On the uniqueness of measuring elastoplastic properties from indentation: The indistinguishable mystical materials. J. Mech. Phys. Solids 55(8), 1618 (2007).

3. N. Choppacoop, M. Dao, and S. Suresh: Depth-sensing instrumented indentation with dual sharp indenters. Acta Mater. 51, 3713 (2003).

4. S. Swaddiwudhipong, K.K. Tho, Z.S. Liu, and K. Zeng: Material characterization based on dual indenters. Int. J. Solids Struct. 42, 69 (2005).

5. J.L. Bucaille, S. Stauss, E. Felder, and J. Michler: Determination of plastic properties of metals by instrumented indentation using different sharp indenters. Acta Mater. 51, 1663 (2003).

6. Y.P. Cao, X.Q. Qian, J. Lu, and Z.H. Yao: An energy-based method to extract plastic properties of metal materials from conical indentation tests. J. Mater. Res. 20(5), 1194 (2005).

7. N. Ogasawara, N. Chiba, and X. Chen: Representative strain of indentation analysis. J. Mater. Res. 20(8), 2225 (2005).

8. L. Wang, M. Ganor, and S.I. Rokhlin: Inverse scaling functions in nanoindentation with sharp indenters: Determination of material properties. J. Mater. Res. 20(4), 987 (2005).

9. D. Tabor: Hardness of Metals (Clarendon Press, Oxford, UK, 1951), pp. 73, 76.

10. K.L. Murty, M.D. Mathew, Y. Wang, V.N. Shah, and F.M. Haggag: Nondestructive determination of tensile properties and fracture toughness of cold worked A36 steel. Int. J. Press. Vessels Pip. 75(11), 831 (1998).

11. J.H. Ahn and D. Kwon: Derivation of plastic stress-strain relationship from ball indentation: Examination of strain definition and pileup effect. J. Mater. Res. 16, 3170 (2001).

12. J.Y. Kim and K.W. Lee: Determination of tensile properties by instrumented indentation technique: Representative stress and strain approach. Surf. Coat. Technol. 201, 4278 (2006).

13. H. Lee: A numerical approach to spherical indentation techniques for material property evaluation. J. Mech. Phys. Solids 53, 203 (2005).

14. S. Jayaraman, G.T. Hahn, W.C. Oliver, C.A. Rubin, and P.C. Bastias: Determination of monotonic stress strain curve of hard materials from ultra-low-load indentation tests. Int. J. Solids Struct. 35, 365 (1998).
15. B. Taljat, T. Zacharia, and F. Kosel: New analytical procedure to determine stress-strain curve from spherical indentation data. Int. J. Solids Struct. 35(33), 4411 (1998).

16. J.S. Field and M.V. Swain: Determining the mechanical properties of small volumes of material from submicron spherical indentations. J. Mater. Res. 10(1), 101 (1995).

17. S. Kucharski and Z. Mroz: Identification of plastic hardening parameters of metals from spherical indentation tests. Mater. Sci. Eng., A 318, 65 (2001).

18. E.G. Herbert, G.M. Pharr, and W.C. Oliver: On the measurement of stress-strain curves by spherical indentation. Thin Solid Films 398-399, 331 (2001).

19. W.P. Yu and J.P. Blanchard: An elastic-plastic indentation model and its solutions. J. Mater. Res. 11(9), 2358 (1996).

20. Y.P. Cao and J. Lu: A new method to extract the plastic properties of metal materials from an instrumented spherical indentation loading curve. Acta Mater. 52(13), 4023 (2004).

21. M.H. Zhao, N. Ogasawara, N. Chiba, and X. Chen: A new approach to measure the elastic-plastic properties of bulk materials using spherical indentation. Acta Mater. 54, 23 (2006).

22. M. Beghini, L. Bertini, and V. Fontanari: Evaluation of the stressstrain curve of metallic materials by spherical indentation. Int J. Solids Struct. 43, 2441 (2006).

23. N. Huber and Ch. Tsakmakis: Determination of constitutive properties from spherical indentation data using neural networks. Part II: Plasticity with nonlinear isotropic and kinematic hardening. J. Mech. Phys. Solids 47, 1589 (1999).

24. A. Nayebi, R. El Abdi, O. Bartier, and G. Mauvoisin: New procedure to determine steel mechanical parameters from the spherical indentation technique. Mech. Mater. 34, 243 (2002).

25. M. Dao, N. Chollacoop, K.J. Van Vliet, T.A. Venkatesh, and S. Suresh: Computational modeling of the forward and reverse problems in instrumented sharp indentation. Acta Mater. 49, 3899 (2001).

26. E.G. Herbert, W.C. Pharr, and G.M. Oliver: On the measurements of yield strength by spherical indentation. Philos. Mag 86(33-35), 5521 (2006).

27. W. Ni, Y.T. Cheng, Z.M. Cheng, and D.S. Grummon: An energybased method for analyzing instrumented spherical indentation experiments. J. Mater. Res. 19, 149 (2004).

28. R.F. Bishop, R. Hill, and N.F. Mott: The theory of indentation and hardness tests. Proc. Phys. Soc. 57(3), 47 (1945).

29. K.L. Johnson: The correlation of indentation experiments. J. Mech. Phys. Solids 18, 115 (1970).

30. X.L. Gao and X.N. Jing: Two new expanding cavity models for indentation deformations of elastic strain-hardening materials. Int. J. Solids Struct. 43, 2193 (2006).

31. R. Hill, B. Storakers, and A.B. Zdunek: A theoretical study of the Brinell hardness test. Proc. R. Soc. London, Ser. A 423, 301 (1989).

32. W.C. Oliver and G.M. Pharr: An improved technique for determining hardness and elastic modulus using load and displacement sensing indentation experiments. J. Mater. Res. 7(6), 1546 (1992).

33. R. Hill: The Mathematical Theory of Plasticity (Oxford University Press, Oxford, London, 1950), p. 97.

34. S.D. Mesarovic and N.A. Fleck: Spherical indentation of elasticplastic solids. Proc. R. Soc. London, Ser. A 455, 2707 (1999).

35. R. Yang, T.H. Zhang, P. Jiang, and Y.L. Bai: Experimental verification and theoretical analysis of the relationships between hardness, elastic modulus, and the work of indentation. Appl. Phys. Lett. 92, 231906 (2008).

36. Y.T. Cheng and C.M. Cheng: Scaling, dimensional analysis and indentation measurements. Mater. Sci. Eng. 44, 91 (2004).

37. E. Meyer: A study on the hardness test. Zeitchrift des Vereines Deutcher Ingenieure. 52, 645 (1908). 